\title{
BMJ Open Mapping mental health recovery tools developed by mental health service users and ex-users: protocol for a scoping review
}

\author{
Hernán María Sampietro, 1,2 Viviana R Carmona (D) ," J Emilio Rojo, ${ }^{3,4}$ \\ Juana Gómez-Benito ${ }^{2,5}$
}

To cite: Sampietro HM, Carmona VR, Rojo JE, et al. Mapping mental health recovery tools developed by mental health service users and ex-users: protocol for a scoping review. BMJ Open 2020;10:e043957. doi:10.1136/ bmjopen-2020-043957

- Prepublication history for this paper is available online. To view these files, please visit the journal online (http://dx.doi. org/10.1136/bmjopen-2020043957).

Received 20 August 2020 Revised 09 0ctober 2020 Accepted 16 October 2020

Check for updates

(c) Author(s) (or their employer(s)) 2020. Re-use permitted under CC BY-NC. No commercial re-use. See rights and permissions. Published by BMJ.

${ }^{1}$ Activament Catalunya Associació, Barcelona, Spain ${ }^{2}$ Department of Social Psychology and Quantitative Psychology, University of Barcelona, Barcelona, Spain ${ }^{3}$ Hospital Benito Menni CASM, Sant Boi de Llobregat, Spain ${ }^{4}$ Department of Psychiatry, International University of Catalunya, Barcelona, Spain ${ }^{5}$ Institute of Neurosciences, University of Barcelona, Barcelona, Spain

Correspondence to Dr Viviana R Carmona; recerca@activament.org

\section{ABSTRACT}

Introduction Since the emergence in 1997 of the Wellness Recovery Action Plan, a number of other tools developed by users and/or ex-users of mental health services have been published and implemented. All these tools aim to promote self-determination in mental health recovery processes. A scoping review will be carried out in order to (1) identify existing tools, (2) describe their distinctive characteristics and (3) examine how they have been implemented and evaluated.

Methods and analysis The scoping review will be guided by the methodological framework proposed by Arksey and 0'Malley and expanded by Levac et al. It will involve, primarily, a literature search of the following electronic databases: Cochrane database, Cumulative Index to Nursing and Allied Health Literature, Psyclnfo, PsycArticles, Scopus, PubMed and Web of Science. In addition, the search process will consider grey literature databases. Users, ex-users and survivors organisations and networks will be contacted in order to identify any relevant material. The reference lists of the articles identified through the literature search will be inspected. Finally, hand searches of journals will be conducted in order to increase the confidence in the search. Two main approaches will be used to present the charted data: a descriptive analysis and a thematic analysis. The study will be performed between April and December 2020. The results will be reported in accordance with the Preferred Reporting Items for Systematic Reviews and Meta-Analyses extension for Scoping Reviews.

Ethics and dissemination This study does not require ethical approval because the data used are from publicly available materials. The study results will be disseminated through an article submitted for publication to a scientific journal and presented at relevant conferences. The results will also be shared in future workshops and seminars as part of continuing education programmes for mental health professionals.

\section{INTRODUCTION}

The recovery model emerged in the 1990s as a result of and response to critiques of psychiatry (notably from the antipsychiatry, survivors and civil rights movements) that ultimately led to deinstitutionalisation. ${ }^{1}{ }^{2}$ A milestone
Strengths and limitations of this study

- All publications that meet the inclusion criteria will be included, regardless of the language in which they are written.

- The review will also consider the manuals and guides that have been developed for each of the recovery plans, not only the publications that evaluate the implementation of these plans.

- The scoping review will include published articles and grey literature. User and ex-user networks will be consulted to obtain any potentially relevant material for inclusion.

in the development of this new model was the publication in 1993 of a paper by William Anthony entitled Recovery from mental illness: the guiding vision of the mental health service system in the 1990s. ${ }^{3}$ After summarising preexisting ideas in the field, Anthony went on to define recovery as: '... a deeply personal, unique process of changing one's attitudes, values, feelings, goals, skills and roles. It is a way of living a satisfying, hopeful and contributing life, even with the limitations caused by illness. Recovery involves the development of new meaning and purpose in one's life as one grows beyond the catastrophic effects of mental illness (p. 527)'.3

During the 1990s, both the USA and New Zealand introduced the recovery model into their policies and practices, and they were followed by the UK and Australia during the early $2000 \mathrm{~s}^{45}$ and by Canada at the end of the same decade. ${ }^{6}$ The same path was followed by several countries in northern Europe, including Germany, Austria, Switzerland, Norway, Sweden and the Netherlands, ${ }^{7}$ as well as by some regional governments in Spain. ${ }^{8}$ This constituted a paradigm shift inspired by international policy on mental health $^{1011}$ and based on the social model of 
disability and the Convention on the Rights of Persons with Disabilities. ${ }^{12}$

In the recovery model the fundamental goal is not the control of symptoms, as in the biomedical model, ${ }^{13} 14$ or the functional adaptation of the person to society, as in the psychiatric/psychosocial rehabilitation model. ${ }^{15}$ Rather, the goal is to enable the person to achieve a meaningful and satisfactory life in accordance with his or her own preferences and values, regardless of the presence of symptoms. ${ }^{316}$ Accordingly, the US Substance Abuse and Mental Health Services Administration defines recovery as a journey of healing and transformation which allows a person to live a satisfying and meaningful life, contributing in a community of her or his choice. ${ }^{17}$ In this model, self-determination and self-management are fundamental factors in the recovery process as they are necessary prerequisites of personal empowerment, of the possibility of regaining control over one's own life. ${ }^{18}$

It was precisely with the aim of promoting selfdetermination in the recovery process of mental health service users that Mary Ellen Copeland developed and published in 1997 the Wellness Recovery Action Plan (WRAP) ${ }^{19}$ The WRAP is a practical tool that helps to identify internal and external resources which may facilitate the recovery of mental health, leading to the creation of an individualised plan for living a satisfying life and achieving well-being. ${ }^{920}$ This tool has been extensively investigated, with studies examining its effects on attitudes and knowledge related to recovery ${ }^{21}$ and its contribution in terms of decreased psychiatric symptoms, ${ }^{22}$ increased hope and quality of life, ${ }^{23}$ a reduced need to use mental health services ${ }^{24}$ and improved propensity for patient self-advocacy. ${ }^{25}$ There is also a systematic review and meta-analysis of the $\mathrm{WRAP}^{26}$ which concludes that its effectiveness lies in its ability to promote self-perceived recovery, rather than to reduce symptoms. All these studies support the use of the WRAP as a tool to promote self-determination in mental health recovery processes.

However, the WRAP is not the only tool that has been designed for this purpose. Two years after the WRAP was first reported, Laurie Ahern and Daniel Fisher published the Personal Assistance in Community Existence (PACE): A Recovery Guide. ${ }^{27}$ The PACE is a training programme aimed at promoting the recovery of people at their own pace. ${ }^{2829}$ According to its authors it is based on the principles of the empowerment model of recovery, that is, selfdetermination, a belief in full recovery, being believed in, trust, respect and non-coercion. ${ }^{28}$

More recently, members of The Icarus Project published another tool: Transformative Mutual Aid Practices (T-MAPs). This was defined as a set of tools that provide space in which to build a personal map of wellness strategies, as well as resilience practices, unique stories and community resources. ${ }^{30}$

What these tools have in common, and which justifies their inclusion in this scoping review, is that they are guides, manuals or practical materials designed to promote self-determination and the active participation of people in their own recovery process. Moreover, because they are based on the experiences of mental health service users and ex-users themselves, they synthesise what these people have learnt during their recovery process. ${ }^{31}$ In addition, although these tools are aimed primarily at people who are experiencing or have experienced a mental disorder, their use is not limited to a specific type of diagnosis or to individuals who have been formally diagnosed. ${ }^{32}$

\section{Study rationale}

Given that the WRAP was published more than 20 years ago and that at least two other tools with a similar purpose have since been created, a scoping review will provide useful information about:

- The existence of tools designed to promote selfdetermination in mental health recovery processes, in addition to the three referred to above.

- Tools of this kind that have been developed beyond the English-speaking world, and/or the adaptation of existing tools to other cultural contexts.

- The distinctive characteristics of available tools, including their similarities and differences.

- The ways in which these tools have been applied, and with what objectives, and how they have been evaluated.

A scoping review is an appropriate way of examining a large set of studies with a wide range of designs, ${ }^{33}$ and it will enable us to gain an overview of the object of study, namely tools developed by mental health service users and ex-users with the aim of promoting recovery, empowerment and well-being. A thematic analysis will be included in the scoping review as a means of interpreting, synthesising and reporting the results. This is an added advantage of the approach that will allow us to better understand how these tools seek to achieve their stated objectives.

\section{Study objectives}

The primary aim is to map the different manuals, guides and similar materials produced by mental health service users and/or ex-users with the aim of promoting recovery, empowerment and well-being in mental health. That is, tools that literally explain that they were created by movements of users and survivors of psychiatry or people with their own experiences in mental health problems and recovery processes. They are based on the knowledge that these experiences provide and not by academic knowledge. The secondary objectives are to explore how these materials have been created, implemented and evaluated, and which are their distinctive features.

\section{METHODS AND ANALYSIS}

Protocol design: We will conduct a scoping review in accordance with the framework described by Arksey and O'Malley, ${ }^{34}$ while also taking into account the recommendations of Levac et $a l^{33}$ This approach will enable 
us to describe the extent, range and nature of evidence in the field. Accordingly, the scoping review will involve five stages: (1) identifying the research question; (2) identifying relevant studies; (3) selecting studies; (4) charting the data; and (5) collating, summarising and reporting the results. The study will be carried out between April and December 2020. The results obtained will be reported following the Preferred Reporting Items for Systematic Reviews and Meta-Analyses extension for Scoping Reviews. ${ }^{35}$

\section{Stage 1: identifying the research question}

Following Arksey and O'Malley's approach, ${ }^{34}$ we will begin by formulating our research question, which will guide the whole of the subsequent literature search and study process. The initial research question will be defined through an iterative process involving all members of the research team. As recommended by Arksey and O'Malley, the question will be formulated in broad terms, focusing on any available tools for promoting the self determination of people in the process of recovering their mental health. In order to clarify this step and to answer the research question, we will define the main concepts, the target population and outcomes of interest for the scoping review. ${ }^{33}$ Therefore, the initial research question that will guide our scoping study will be: which tools, created by people with direct experiences of mental health problems and personal recovery and/or movements of users, ex-users and survivors of psychiatry, exist to develop personal recovery plans and to promote self-management of well-being? This question will allow us to identify and explore the specific characteristics of the tools, including how they have been created and implemented.

\section{Stage 2: identifying relevant studies}

This stage will involve the identification of different sources for answering our research question, as well as the creation of search strategies for identifying studies. The search strategies will be updated and refined based on abstracts retrieved from the initial searches. ${ }^{33}$ We will use three main strategies to ensure the inclusion of all relevant studies. First, we will carry out searches in the following databases: Cochrane database, Cumulative Index to Nursing and Allied Health Literature, PsycInfo, PsycArticles, Scopus, PubMed and Web of Science without date and language restrictions. These databases have been chosen for their broad coverage of the discipline and of topics such as wellness, recovery, mental health and mental disorder. The search terms from the following keywords: action plan, crisis plan, crisis program, empowerment, making choice, mapping madness, Mapping Our Madness, own pace, Personal Assistance in Community Existence, taking action, Transformative mutual aid practices, self-determination, self-management, recovery program, wellness, wellness recovery action plan, wellness recovery action planning will be useful to capture any potential resources from the databases. Boolean operators (AND, OR, NOT) will be used to combine them. The initial search strategy already used in the Scopus database is as follows: ("Wellness Recovery Action Plan*" OR "Personal Assistance in Community Existence" OR "Mapping Our Madness" OR "Transformative mutual aid practices") in topic OR ("empower*" OR "self-determination" OR "wellness" OR "self manag*" OR "self-manag*" OR "get better" OR "mak* choice*" OR "tak* action") in title AND ("crisis plan*" OR "action plan*" OR "action program*” OR "crisis program*” OR "recovery plan*" OR "recovery program*" OR "mapping mad" OR "own pace") in topic. Grey literature databases such as EThOS and the System for Information on Grey Literature in Europe will also be considered in the search process. Because of the specific features of grey literature databases, each of the following keywords will be entered separately without language or year of publication restriction: Wellness Recovery Action Plan, Personal Assistance in Community Existence, Mapping Our Madness, Transformative mutual aid Practices, T-MAPS, Madness and Oppression. Only the most relevant titles will be extracted and assessed for possible inclusion.

Second, mental health experts by experience and organisations and networks working in this area will be contacted in order to locate any relevant study or material. The worldwide network of users, ex-users and survivors of psychiatry is interconnected and maintains fluid communication. Moreover, the authors (HMS and VRC) are also members of a users' association, ActivaMent Catalunya Associació, which is part of several networks such as: (1) The Latin American and Caribbean Network of Human Rights and Mental Health; (2) Absolute Prohibition-Campaign to support the Convention on the Rights of Persons with Disabilities absolute prohibition of commitment and forced treatment. The association also maintains close connections with entities such as: (3) World Network of Users and Survivors of Psychiatry; (4) European Network of Users and Survivors of Psychiatry. These previous established connections will facilitate and make possible to identify any material of interest.

Third, we will screen the reference lists of articles identified through the literature search and conduct hand searches of journals in order to identify any additional relevant studies and to achieve the level of comprehensiveness required. Finally, an additional search with no date restriction will be also conducted in Google Scholar (only the first 100 outcomes will be inspected). The search strategy in this case will be the same as the one used with grey literature databases.

All records obtained from the search process will be evaluated for possible inclusion regardless of the language in which they have been published. And if necessary, expert translators for Chinese, Korean, Dutch, Arab and Japanese will be available.

\section{Stage 3: study selection}

The results obtained from each search will be downloaded into a citation management database, where duplicate records will be removed. We will use a two-stage 
study selection process comprising (1) screening of titles and abstracts, and (2) a full-text review.

Following the recommendations made by Levac et $a l^{33}$ two reviewers will independently inspect titles, abstracts and full-texts to identify potentially relevant studies. All articles considered relevant by either reviewer will be included for full-text evaluation. Any disagreements in the selection process will be resolved by discussion with a third reviewer. The inclusion and exclusion criteria will be drawn up by consensus among the research team and tested on a sample of abstracts to determine whether they capture studies with the potential to answer the research question. Research papers, conference abstracts, dissertations, books and book chapters, manuals, guides and research reports will be considered for inclusion if:

a. They concern materials or practical tools for promoting self-determination and empowerment of people in their mental health recovery process.

b. They report tools based on the recovery model.

c. They are comprehensive tools for improving wellbeing and recovery (more than just a crisis plan or a wellness toolbox).

d. They are tools developed by people who are experiencing or have experienced a mental health issue and/or by users, ex-users and survivors' movements.

e. They are tools developed for use by people who experience psychiatric symptoms, although they can be used by any person who wishes to achieve health and well-being.

f. They are studies involving participants aged 18+.

g. They are publications reporting the main applications of tools in people with any mental health problem.

Exclusion criteria will mainly concern the study design, and hence opinion articles, reviews, protocols and metaanalyses will not be considered for inclusion.

Since quality assessment does not take part of the scoping study methodology because it is not addressed to assess the evidence for a particular intervention, ${ }^{34}$ we will not consider the quality of studies as a criterion for inclusion/exclusion. However, the integration of multiple documents and research designs requires the use of a set of criteria to choose the best evidence. In our study, this set has the following criteria: (1) when there are multiple documents reporting the same target with the same participants, only the published articles will be considered for inclusion. (2) In cases of reports describing the implementation or assessment of a tool, only the documents with some basic information regarding study (year of publication, authors, country, sample size, design, evaluated tool, main results) and participants' characteristics (gender, age, profile: users, professionals, family or relatives) will be considered for inclusion. (3) Regarding specific tools localised through the network of users, ex-users and survivors of psychiatry, the quality criteria will be their application and recognition as useful tools for people integrating these networks.

\section{Stage 4: charting the data}

A preliminary scoping phase has already allowed us to develop a data extraction framework comprising 39 categories (see table 1). This framework represents an initial consensus about what is relevant to record, and it will be used to review every retrieved text that fulfils the criteria for inclusion.

Two team members will pilot the data extraction framework using a sample comprising $10 \%$ of the included documents, the aim being to demonstrate that the framework is being applied consistently. Any disagreements will be discussed and resolved by consensus among all team members. If necessary, the framework will be revised, any ambiguous categories changed and categories that come up during the data extraction process will be discussed and added to the data extraction form. The data from each included document will likewise be charted by two team members working independently. Any discrepancies in the extracted data will be resolved by arbitration in a consensus meeting.

Stage 5: collating, summarising and reporting the results Following recommendations by Arksey and O'Malley, ${ }^{34}$ two main approaches will be used to present the charted data: a descriptive analysis and a thematic analysis. Descriptive analysis will involve numerical calculations of frequency in order to display the extent, nature and distribution of studies included in the scoping review. Specifically, we will carry out a descriptive analysis focused on country, type of study, design, data analysis and synthesis, the demographics characteristics of the sample and the characteristics of the tools identified. These initial descriptive analyses will identify the predominant research methods and the geographical locations in the literature. Thematic analysis will be performed; that is, the articles included will be organised and analysed by recurring themes, points of agreement and points of disagreement. ${ }^{34} 3637$ That is to identify, examine and record the relevant patterns or themes in order to answer our research question.

\section{ETHICS AND DISSEMINATION}

This scoping review will be the first study to compare different tools for developing personal recovery and wellness plans in mental health, specifically those based on the recovery model and created by mental health service users and ex-users themselves. The results obtained will be useful in terms of adapting existing tools and developing new tools for different cultural contexts.

This protocol is based on a comprehensive, rigorous and transparent methodology. It does not require ethical approval because the scoping review methodology involves collecting and categorising publicly available materials.

The results of the scoping review will be disseminated through an article submitted for publication to a scientific journal and presented at relevant conferences. They 
Table 1 Data extraction framework

N Category Description

Descriptive characteristics of the document

1 Type of document The categories are: (a) paper, (b) guide, (c) manual, (d) book chapter (e) book and (f) other.

2 DOI Digital Object Identifier

3 Database Database from which the document was extracted. If the document is identified by consulting reference lists, record the first author and the year of publication (eg, Copeland, 1997). If the document is retrieved through a manual search, record the name of the journal inspected and the search date (eg, BMJ Open 20200818).

\begin{tabular}{|c|c|c|}
\hline 4 & Year & Year of publication \\
\hline 5 & Authors & Authorship of the document \\
\hline 6 & Title & Title of the document \\
\hline 8 & Aim of the tool & $\begin{array}{l}\text { What is the purpose of the tool described in the document? }{ }^{*} \text { This category is only applicable for original } \\
\text { documents or manuals in which the creation of the tool is explained. }\end{array}$ \\
\hline 9 & $\begin{array}{l}\text { Publication (Journal/ } \\
\text { Book) }\end{array}$ & $\begin{array}{l}\text { Record where the document has been published (eg, record name of journal for a paper, name of book for } \\
\text { a book chapter). In the case of an unpublished document, record as 'unpublished'. }\end{array}$ \\
\hline 10 & Country & $\begin{array}{l}\text { Name of the country or countries participating in the publication. If there are two or more authors from the } \\
\text { same country, record as a single contribution from that country. }\end{array}$ \\
\hline 11 & Institution & The name of the authors' institution and/or intellectual property of the document. \\
\hline 12 & Language & Language in which the documents have been written. \\
\hline
\end{tabular}

Study methodology

\begin{tabular}{|c|c|c|}
\hline 13 & Aim & Aim or purpose of the study \\
\hline 14 & Type of objective & $\begin{array}{l}\text { Record the purpose of the study. The categories are: (a) construction of the tool, (b) exploration, (c) } \\
\text { evaluation, (d) other (eg, reporting/description, argumentation in favour). }\end{array}$ \\
\hline 15 & Study design & $\begin{array}{l}\text { Code the design of the study. The categories are: (a) RCT, (b) non-randomised controlled trial, (c) pretest/ } \\
\text { post-test design, (d) qualitative design, (e) mixed methods, (f) other. }\end{array}$ \\
\hline 16 & Outcomes measured & $\begin{array}{l}\text { Specify what was investigated in the study (eg, satisfaction, stage of the recovery process, empowerment, } \\
\text { symptoms, etc.). }\end{array}$ \\
\hline 18 & Data analysis & Record the techniques and/or theories used to gather and interpret the data. \\
\hline 19 & $\begin{array}{l}\text { Instrument(s) for } \\
\text { assessment }\end{array}$ & If interventions have been evaluated, specify which evaluation instruments were used in the study. \\
\hline 20 & Control group & If the study includes a comparison/control group, specify its composition (sample characteristics). \\
\hline 21 & Who evaluates & $\begin{array}{l}\text { Record who evaluates what is measured or collected as data. The categories are: (a) professional } \\
\text { evaluation (by academic or service professionals), (b) first-person evaluation (ie, by the persons evaluated } \\
\text { themselves and/or by other users of mental health services), (c) mixed, that is, both (a) and (b). }\end{array}$ \\
\hline
\end{tabular}

\section{Characteristics of participants}

23 Type of participants

\begin{tabular}{|c|c|c|}
\hline 24 & Sample size & $\begin{array}{l}\text { Number of people participating in the study (either in the process of constructing the tool or the sample } \\
\text { size in studies that evaluate a programme). Specify the number of users, professionals and/or family } \\
\text { members whenever possible. }\end{array}$ \\
\hline 25 & Diagnosis & $\begin{array}{l}\text { Record whether or not the document specifies a diagnosis for the people participating in the study (eg, } \\
\text { yes, no). Code diagnoses if they are stated in the document. }\end{array}$ \\
\hline 26 & Age & Age of participants. Indicate mean and SD in brackets(eg, 24 years (6.5)). \\
\hline 27 & Education & Number of years of education. Report mean and SD(eg, 10 (2.3)). \\
\hline
\end{tabular}




\begin{tabular}{|c|c|c|}
\hline $\mathbf{N}$ & Category & Description \\
\hline 30 & Other & $\begin{array}{l}\text { Other descriptive variables regarding participants. Code any other variable of potential interest for the } \\
\text { analysis (eg, marital status, employment status, etc). }\end{array}$ \\
\hline \multicolumn{3}{|c|}{ Implementation } \\
\hline 31 & Access & Record whether or not the tool is open access (eg, yes, no). \\
\hline 32 & Type of application & $\begin{array}{l}\text { Indicate how the tool is applied: (a) individual, (b) group. If it is applied individually, record in brackets if the } \\
\text { tool can be self-applied, with or without external support (eg, individual (self-applied)). In the case of group } \\
\text { application, specify in brackets if this was through a course, workshop or both(eg, group (course)). }\end{array}$ \\
\hline 33 & Duration & Whenever possible, specify the duration of the programme or number of sessions. \\
\hline 34 & Setting & Location in which the programme has been implemented or the study carried out. \\
\hline 35 & Activities & $\begin{array}{l}\text { Record the activities performed in the workshops or sessions used for implementation of the tool (eg, life } \\
\text { stories, participatory dynamics, MAG, etc.). }\end{array}$ \\
\hline 36 & Trainers & $\begin{array}{l}\text { In the case of workshops/training, indicate who the trainers are. Specify if the training is given: (a) Through } \\
\text { peer support alone, (b) Through peer support plus one or more non-peer professionals, (c) By one or more } \\
\text { non-peer professionals, (d) Not specified. }\end{array}$ \\
\hline 37 & $\begin{array}{l}\text { Support material for } \\
\text { implementation of } \\
\text { recovery plan }\end{array}$ & $\begin{array}{l}\text { Indicate whether or not the tool serves as support material to implementation of a recovery plan or } \\
\text { recovery strategy (eg, yes, no). It can be an independent workbook or a section of the material with } \\
\text { questions to answer. Specify in brackets the type of support material (eg, yes (workbook)). }{ }^{*} \text { This category is } \\
\text { only applicable for original documents or manuals in which the creation of the tool is explained. }\end{array}$ \\
\hline 38 & $\begin{array}{l}\text { Support material for } \\
\text { implementation of } \\
\text { workshops/ training }\end{array}$ & $\begin{array}{l}\text { Indicate whether or not the tool serves as support material to the implementation of workshops or training } \\
\text { (eg, yes, no). It can be a guide, a manual and/or teaching material showing the content and structure of the } \\
\text { sessions. Specify in brackets the type of support material(eg, yes (guide)). }{ }^{*} \text { This category is only applicable } \\
\text { for original documents or manuals in which the creation of the tool is explained. }\end{array}$ \\
\hline 39 & $\begin{array}{l}\text { Follow-up support } \\
\text { material }\end{array}$ & $\begin{array}{l}\text { Indicate whether or not the tool serves as support material to the follow-up and assessment of the } \\
\text { implementation of, compliance with and/or usefulness of the recovery plan in question (eg, yes, no). It can } \\
\text { be independent material and/or a section included in the materials. Specify in brackets the type of support } \\
\text { material (eg, yes (workbook)). }{ }^{*} \text { This category is only applicable for original documents or manuals in which } \\
\text { the creation of the tool is explained }\end{array}$ \\
\hline
\end{tabular}

MAG, mutual aid group; RCT, randomised controlled trial.

will also be shared in workshops and seminars offered by the authors as part of their ongoing contribution to the training of future professionals in nursing, clinical psychology and psychiatry, as well as in continuing education programmes for mental health professionals.

\section{Patient and public involvement}

The manuscript has been designed and developed by users of mental health services. The fact that the research team includes them is an added advantage when it comes to identifying tools, and it will also help to contextualise the findings. The knowledge produced by the scoping review will be useful for various key stakeholders within health systems.

Contributors HMS and VRC designed the study and the initial search strategy. HMS developed the conceptual framework and wrote the first draft of the manuscript. VRC developed the methodological framework and reviewed the draft of the manuscript. JG-B and JER provided regular feedback on each of these steps and contributed to revision of the final manuscript.

Funding This work was supported by Spain's Ministry of Economy and Competitiveness (grant number PSI2015-67984-R) and the Catalan Agency for the Management of University and Research Grants (grant number 2017SGR1681)

\section{Competing interests None declared.}

Patient and public involvement Patients and/or the public were involved in the design, or conduct, or reporting, or dissemination plans of this research. Refer to the Methods section for further details.

Patient consent for publication Not required.
Provenance and peer review Not commissioned; externally peer reviewed.

Open access This is an open access article distributed in accordance with the Creative Commons Attribution Non Commercial (CC BY-NC 4.0) license, which permits others to distribute, remix, adapt, build upon this work non-commercially, and license their derivative works on different terms, provided the original work is properly cited, appropriate credit is given, any changes made indicated, and the use is non-commercial. See: http://creativecommons.org/licenses/by-nc/4.0/.

ORCID iD

Viviana R Carmona http://orcid.org/0000-0002-0525-0987

\section{REFERENCES}

1 Braslow JT. The manufacture of recovery. Annu Rev Clin Psychol 2013;9:781-809.

2 Alonso Suárez M. Reflexiones sobre El modelo de recuperación en salud mental. Boletín la Asoc Madrileña Salud Ment 2016;40:14-22.

3 Anthony WA. Recovery from mental illness: the guiding vision of the mental health service system in the 1990s. Psychosoc Rehabil J 1993;16:11-23.

4 Jacobson N, Curtis L. Recovery as policy in mental health services: strategies emerging from the states. Psychiatr Rehabil J 2000;23:333-41.

5 Ramon S, Healy B, Renouf N. Recovery from mental illness as an emergent concept and practice in Australia and the UK. Int J Soc Psychiatry 2007;53:108-22.

6 Piat M, Sabetti J, Couture A, et al. What does recovery mean for me? perspectives of Canadian mental health consumers. Psychiatr Rehabil J 2009;32:199-207.

7 Slade M, Amering M, Oades L. Recovery: an international perspective. Epidemiol Psichiatr Soc 2008;17:128-37.

8 Generalitat de Catalunya. Pla integral d'atenció a les persones amb trastorn mental $i$ addiccions 2017-2019. Generalitat de Catalunya, 2017: $41 p$ 
9 de CG. Estratègies 2017-2020 Pla director de salut mental i addiccions [online]. Departament de Salut, 2017: 84. http:// salutweb.gencat.cat/ca/ambits_tematics/linies_dactuacio/ estrategies_salut/salut_mental_i_addiccions/\%0Ahttp://salutweb. gencat.cat/web/.content/home/ambits_tematics/linies_dactuacio/ salut_i_qualitat/plans_directors/salut_mental_i_addiccions/que_ es/d

10 WHO. Mental health action plan 2013-2020. World Health Organization, 2013: 50.

11 WHO - Regional Office for Europe. The European Mental Health Action Plan 2013-2020 [online. World Health Organization, 2013: 26. http://www.euro.who.int/_data/assets/pdf_file/0020/280604/WHOEurope-Mental-Health-Acion-Plan-2013-2020.pdf

12 United Nations. Convention on the rights of persons with disabilities. United Nations, 2006: 37

13 Wade DT, Halligan PW. Do biomedical models of illness make for good healthcare systems? BMJ 2004;329:1398-401.

14 Deacon BJ. The biomedical model of mental disorder: a critical analysis of its validity, utility, and effects on psychotherapy research. Clin Psychol Rev 2013;33:846-61.

15 Bond GR, Resnick SG. Psychiatric rehabilitation. In: Frank RG, Elliott TR, eds. Handbook of rehabilitation psychology. American Psychological Association, 2000: 235-58.

16 Shepherd G, Boardman J, Slade M. Making recovery a reality. Scottish recovery network [online], 2008: 1-23. http://www.scmh.org. uk/pdfs/Recovery_lived_experience_perspective.pdf

17 SAMHSA. Recovery for me mental health services: practice guidelines for Recovery-Oriented care. Substance Abuse and Mental Health Services Administration, 2011.

18 Copeland ME. Self-determination in mental health recovery: taking back our lives. In: Jonikas JA, Cook JA, eds. National selfdetermination \& psychiatric disability invitational conference [online]. Chicago, IL: University of Illinois, 2004: 68-82. https://www.theweb. ngo/history/recovery/UICsdconfdoc19.pdf\#page $=74$

19 Copeland ME. Wellness recovery action plan. Peach Press, 1997.

20 Copeland ME, Jonikas JA. Wellness recovery action planning: the role of wellness promotion in a new paradigm of community mental health. In: Nelson G, Kloos B, Ornelas J, eds. Community psychology and community mental health: towards transformative change. Oxford University Press, 2012: 133-51.

21 Doughty C, Tse S, Duncan N, et al. The wellness recovery action plan (WRAP): workshop evaluation. Australas Psychiatry 2008;16:450-6.
22 Fukui S, Starnino VR, Susana M, et al. Effect of wellness recovery action plan (wrap) participation on psychiatric symptoms, sense of hope, and recovery. Psychiatr Rehabil J 2011;34:214-22.

23 Cook JA, Copeland ME, Floyd CB, et al. A randomized controlled trial of effects of wellness recovery action planning on depression, anxiety, and recovery. Psychiatr Serv 2012;63:541-7.

24 Cook JA, Jonikas JA, Hamilton MM, et al. Impact of wellness recovery action planning on service utilization and need in a randomized controlled trial. Psychiatr Rehabil J 2013;36:250-7.

25 Jonikas JA, Grey DD, Copeland ME, et al. Improving propensity for patient self-advocacy through wellness recovery action planning: results of a randomized controlled trial. Community Ment Health J 2013;49:260-9.

26 Canacott L, Moghaddam N, Tickle A. Is the wellness recovery action plan (wrap) efficacious for improving personal and clinical recovery outcomes? A systematic review and meta-analysis. Psychiatr Rehabil J 2019;42:372-81.

27 Ahern L, Fisher D. Personal assistance in community existence. A recovery guide. National Empowerment Center, Inc, 1999: 1-35.

28 Fisher DB, Ahern L. Personal assistance in community existence (PACE): an alternative to PACT. Ethical Hum Sci Serv 2000;2:87-92.

29 Ahern L, Fisher D. Recovery at your own PACE. J Psychosoc Nurs Ment Health Serv 2001;39:22-32.

30 McNamara J, DuBrul SA. T-MAPs. Transformative mutual aid practices [online], 2018: 36. https://tmapscommunity.net/

31 Copeland ME. Action planning for prevention and recovery: a selfhelp guide. recovering your mental health series. Subst Abus Ment Heal Serv Adm [online] 2002;10:42.

32 Copeland ME. Wellness recovery action plan: a system for monitoring, reducing and eliminating uncomfortable or dangerous physical symptoms and emotional feelings. Occup Ther Ment Heal 2002;17:127-50.

33 Levac D, Colquhoun H, O'Brien KK. Scoping studies: advancing the methodology. Implementation Sci 2010;5:1-9.

34 Arksey H, O'Malley L. Scoping studies: towards a methodological framework. Int J Soc Res Methodol 2005;8:19-32.

35 Tricco AC, Lillie E, Zarin W, et al. PRISMA extension for scoping reviews (PRISMA-ScR): checklist and explanation. Ann Intern Med 2018;169:467-73.

36 Braun V, Clarke V. Using thematic analysis in psychology. Qual Res Psychol 2006;3:77-101.

37 Clarke V, Braun V. Thematic analysis. J Posit Psychol 2017;12:297-8. 